\title{
New insights in understanding the pathogenesis of spondyloarthropathies
}

\author{
Irene E van der Horst-Bruinsma*1 and J Bart A Crusius² \\ See related research by Sharma et al., http://arthritis-research.com/content/11/6/R168
}

\begin{abstract}
Spondyloarthropathies (SpA) are characterised by dysregulation of the inflammatory processes and bone metabolism which may be clarified by gene expression profiles. Sharma and colleagues showed associations of axial SpA with the innate immune system, inflammation markers and markers of bone remodeling. Drawbacks of this study are the patient selection based on uveitis, which limits the extrapolation of these data, and the racial difference between index cases and controls, which contributes to differences in gene expression. Nevertheless, this study provides a direction for unraveling the intriguing balance between inflammation and ossification in ankylosing spondylitis.
\end{abstract}

Gene expression profiles may clarify our understanding of the pathophysiology of disease, and by redefining pathways of inflammation may offer new alternatives for treatment of chronic diseases such as the spondyloarthropathies (SpA). If the expression profiles are specific for the disease or for a particular subgroup of patients, the data may generate biomarkers to classify the disease and define the stage of the inflammatory process. Genome-wide RNA expression analysis will thus lead to new insights in the pathogenesis of heterogeneous diseases such as SpA. These studies are expensive and powerful statistical approaches are necessary for achieving reproducible results. The potential gains, however, are of great clinical importance. Attention should be given to the selection of patients, to the state of the inflammatory process and to the possible effect of

\footnotetext{
*Correspondence: IE.vanderHorst@vumc.nl

'Department of Rheumatology, VU University Medical Centre, PO Box 7057,

1007 MB, Amsterdam, The Netherlands

Full list of author information is available at the end of the article
}

current therapy in the enhancing or silencing of this process.

As far as we know, only two studies have used whole genome expression profiles in blood from SpA patients and controls. Both studies follow a similar protocol; the study published in the previous issue of Arthritis Research and Therapy used an Affymetrix microarray system [1], and an Australian study published online in Annals of the Rheumatic Diseases used an Illumina Chip [2]. Both studies use a primary set and a validation set. Both studies also use peripheral blood, and obviously the pathogenesis of joints may be slightly different when the organ of disease is targeted. The approach, however, is valid.

The study described by Sharma and colleagues showed several interesting associations of axial SpA with the innate immune system - like the NOD-like receptor family member inflammasome component NLRP2, secretory leukocyte peptidase inhibitor I, and secreted protein acidic, cysteine-rich (also known as osteonectin) and with inflammation markers (IL-1 receptors) and markers of bone remodeling (Kringle containing transmembrane protein 1 (KREMEN1)) [1]. Limitations of this study are the patient selection, which was based on uveitis, and that neither joint assessment nor radiographic data were included to confirm the diagnosis of ankylosing spondylitis (AS). The more correct diagnosis of axial SpA was therefore used. Unfortunately this patient selection limits the extrapolation of these data to AS or SpA.

Another important issue is the high number of females in the control group ( $80 \%$ in the combined sets) compared with the SpA subjects (28\%). It is not yet known whether gender differences may have influenced the mRNA expression profiles, and moreover whether this difference was taken into account with the linear models. More important is the racial difference between index cases (22\% Asian) and controls (92\% Caucasian, 8\% nonCaucasian) because up to $25 \%$ of the gene expression can be attributed to these racial differences [3]. This was not corrected in the linear models and might account for 
some of the differences in expression profiles between uveitis patients and controls.

The results of this study in SpA patients by Sharma and colleagues, although interesting, should therefore await validation with a similar group of selected patients. Some of these transcripts are related to genes previously described to be associated with AS (such as the IL-1 family, and triggering receptor expressed on myeloid cells-like 1) but others (such as secretory leukocyte peptidase inhibitor I and secreted protein acidic, cysteinerich) are new. Secretory leukocyte peptidase inhibitor I, which downregulates TNF, could be an interesting target for future studies. Secreted protein acidic, cysteine-rich associated with scleroderma - suggests an association with dysregulation of the fibrous tissue that might also be involved in the pathogenesis of AS. The most promising aspects for future research derive from the markers of bone remodeling - such as KREMEN1, which might influence the wnt-signaling pathway. These markers can give direction towards further larger studies with a proper definition of patients and follow up unraveling the intriguing balance between inflammation and ossification in the pathogenesis of AS.

The classification, genetic susceptibility, pathology, and response to treatment of spondyloarthritis have been evaluated into five interrelated subsets - namely, AS, psoriatic arthritis, reactive arthritis, arthritis associated with inflammatory bowel disease, and undifferentiated spondyloarthropathy - and have been reviewed, as have the classification criteria so far used [4]. The data presented by Sharma and colleagues in the previous issue of the journal and the previously quoted Australian study support that SpA as well as other autoimmune diseases will be better characterised at the level of gene expression. It is to be hoped that the understanding of the different pathways to inflammation will facilitate the assessment of disease activity and will improve targeting of therapies [5].

\section{Abbreviations}

AS = ankylosing spondylitis; $\mathrm{L}$ = interleukin; SpA = spondyloarthropathies; TNF = tumor necrosis factor.

\section{Author details}

'Department of Rheumatology, VU University Medical Centre, PO Box 7057, 1007 MB, Amsterdam, The Netherlands

2Laboratory of Immunogenetics, Department of Pathology, VU University

Medical Centre, PO Box 7057, 1007 MB, Amsterdam, The Netherlands

\section{Competing interests}

The authors declare that they have no competing interests.

Published: 18 January 2010

\section{References}

1. Sharma SM, Choi D, Planck SR, Harrington CA, Austin CR, Lewis JA, Diebel TN, Martin TM, Smith JR, Rosenbaum JT: Insights in to the pathogenesis of axial spondylartropathy based on gene expression profiles. Arthritis Res Ther 2009, 11:R168.

2. Duan R, Leo P, Bradbury L, Brown MA, Thomas GP: Gene expression profiling reveals a down-regulation in immune-associated genes in AS patients. Ann Rheum Dis 2009. [Epub ahead of print: doi:10.1136/ard.2009.111690]

3. Couzin J: Human genetics. In Asians and whites, gene expression varies by race. Science 2007, 315:173-174.

4. Davis JC, Jr, Mease PJ: Insights into the pathology and treatment of spondyloarthritis: from the bench to the clinic. Semin Arthritis Rheum 2008, 38:83-100.

5. Baechler EC, Batliwalla FM, Reed AM, Peterson EJ, Gaffney PM, Moser KL, Gregersen PK, Behrens TW: Gene expression profiling in human autoimmunity. Immunol Rev 2006, 210:120-137.

doi:10.1186/ar2895

Cite this article as: van der Horst-Bruinsma IE, Crusius JBA: New insights in understanding the pathogenesis of spondyloarthropathies. Arthritis Research \& Therapy 2010, 12:102. 